\title{
Tratamento do aneurisma de arco e aorta descendente: abordagem cirúrgica em uma etapa
}

Single stage surgical approach for arch and descending thoracic aorta aneurysm

Simone Alves DANTAS, Ricardo Ribeiro DIAS, Noedir A. G. STOLF, Sérgio Almeida de OLIVEIRA

RBCCV 44205-735

\section{Resumo}

Paciente de 61 anos procurou atendimento médico com quadro clínico de infecção pulmonar. $O$ estudo radiológico de tórax revelou aneurisma de aorta descendente com comprometimento do arco aórtico. Ressalta-se a correção cirúrgica em uma única etapa, por meio de abordagem operatória não convencional, que possibilitou a correção total do aneurisma.

Descritores: Aorta, cirurgia. Aneurisma da aorta torácica, cirurgia.
Abstract

We report on a 61-years-old male patient, who had an extensive arch and descending thoracic aorta aneurysm diagnosed while treating a pulmonary infection. The objective of this work is to report an unusual single stage surgical approach to correct an extensive arch and descending thoracic aorta aneurysm.

Descriptors: Aorta, surgery. Aortic aneurysm, thoracic, surgery.

Trabalho realizado no Instituto do Coração (InCor) - HC-FMUSP

Endereço para correspondência: Simone A. Dantas. Instituto do Coração (InCor) - HC-FMUSP (Unidade Clínica de Miocardiopatias). Av.Dr.Enéas de Carvalho. Aguiar,44. São Paulo -SP. CEP 05403900. Tel: (11) 3069-5057. Fax: (11) 3069-5346.

Artigo recebido em novembro de 2004

E-mail: simonedantas@cardiol.br 


\section{INTRODUÇÃO}

Aneurisma de aorta torácica descendente é a causa mais comum de doença da aorta torácica que necessita de intervenção cirúrgica. A principal etiologia é a doença aterosclerótica, podendo ser secundários também a trauma torácico fechado, infecção ou doenças do tecido conectivo [1]. Apresentam crescimento anual médio em torno de $0,10 \mathrm{~cm} /$ ano [2] e pela sua evolução silenciosa podem apresentar risco operatório adicional, uma vez que o diagnóstico, muitas vezes, é feito quando a doença já se encontra em fase avançada ou secundariamente a complicações, como ruptura e dissecção.

O objetivo deste relato é apresentar a tática operatória com abordagem única para a correção de grande aneurisma localizado no arco aórtico e na aorta descendente, cuja abordagem tradicional, por meio de esternotomia ou toracotomia esquerda, não permitiria o tratamento da lesão existente numa única etapa cirúrgica.

\section{RELATO DOCASO}

Paciente de 61 anos, masculino, pardo, ex-tabagista, procurou assistência médica com quadro de febre, dispnéia leve e dor torácica atípica. O exame físico foi inespecífico, porém o estudo radiológico simples de tórax evidenciou acentuado alargamento do mediastino. A tomografia helicoidal da aorta torácica permitiu completa avaliação do aneurisma, justificando a intervenção cirúrgica.

\section{Procedimento cirúrgico}

A bitoracotomia ântero-lateral pelo $4^{\circ}$ espaço intercostal foi realizada com paciente em decúbito dorsal levemente inclinado para direita, possibilitando o acesso a todo mediastino e ao arco aórtico, até o terço distal da aorta descendente (Figura 1A).

A instalação da circulação extracorpórea foi realizada pela artéria subclávia direita e a drenagem venosa por meio de cânula única em átrio direito. A proteção cerebral, durante o período de parada circulatória total, foi obtida por meio de hipotermia profunda $\left(18^{\circ} \mathrm{C}\right)$ associada ao resfriamento tópico da cabeça com gelo e a perfusão anterógrada seletiva pela artéria carótida direita. Dessa forma, foi possível a interposição de tubo de dacron da aorta ascendente à porção distal da aorta descendente, com o reimplante dos vasos da base no tubo (Figura 1B e C).

No período pós-operatório, o paciente apresentou dificuldade na retirada da assistência respiratória, por distúrbio ventilatório secundário a paralisia frênica esquerda, decorrente de lesão iatrogênica pelo extenso comprometimento da dilatação. Foi extubado no $5^{\circ}$ dia de pós-operatório sem outras intercorrências, recebendo alta hospitalar no $15^{\circ}$ dia.

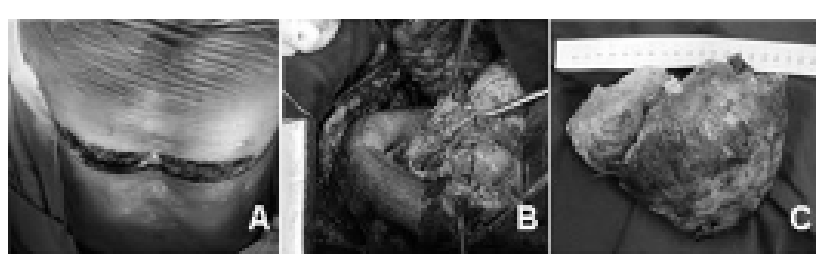

Fig. 1 - A - Aspecto intra-operatório da incisão cirúrgica da bitoracotomia; B - Fotografia intra-operatória do aspecto final da substituição cirúrgica da aorta descendente, arco e parte da aorta ascendente com o reimplante dos vasos da base; $\mathrm{C}$ - Tamanho do trombo intraluminal envolvido no aneurisma.

A tomografia helicoidal de tórax, no controle tardio, mostrou resolução completa da doença de base (Figura 2B), corrigindo a alteração constatada na radiografia inicial (Figura 2A).

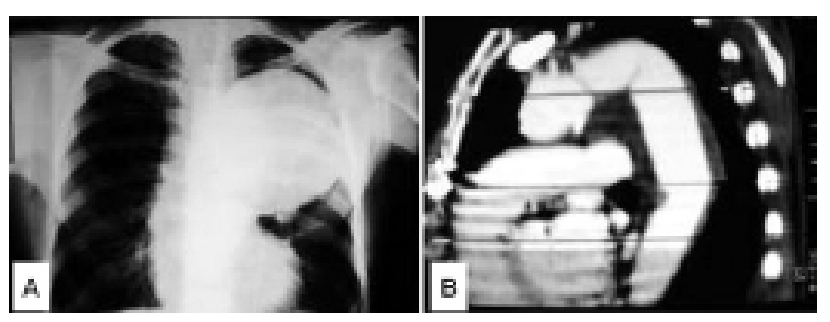

Fig. 2 - A - Radiografia de tórax realizada no período pré-operatório que mostra grande tumoração no mediastino médio e superior, invadindo hemitórax esquerdo. B - Tomografia helicoidal de aorta torácica, demonstrando o aspecto final da aorta torácica reconstruída.

\section{COMENTÁRIOS}

Os aneurismas de aorta descendente, em função da sua localização anatômica no tórax, tendem a evoluir de forma assintomática e, quando presentes, os sintomas estão relacionados à rotura, à dissecção ou ao acometimento de estruturas adjacentes. Os pacientes podem apresentar disfonia - quando há acometimento do nervo laríngeo recorrente, insuficiência respiratória, episódios hemoptóicos ou hemoptise secundários ao comprometimento pulmonar e disfagia ou hematêmese no comprometimento esofágico. Sua incidência de 10 casos por 100.000 habitantes/ano [1] tende a aumentar, em virtude do aumento da expectativa de vida da população brasileira.

A evolução lenta permite crescimento até grandes proporções, sem a presença de diagnóstico. A indicação cirúrgica dependerá dos sintomas ou do diâmetro transversal da aorta e/ou da sua velocidade de crescimento quando assintomáticos [2].

A técnica operatória de abordagem da aorta através da bitoracotomia ântero-lateral com esternotomia transversa 
foi recentemente apresentada por KOUCHOUKOS et al. [3] em pacientes submetidos à reoperação para correção de dissecção de aorta tipo A de Stanford. Segundo os autores, a técnica proporciona melhor exposição do segmento dilatado do arco aórtico, aorta descendente e vasos da base.

Quando o aneurisma não compromete o arco aórtico, é possível a abordagem de extensos aneurismas através da toracotomia esquerda ou da toracofrenolaparotomia, com técnicas semelhantes de proteção à lesão isquêmica da medula e cerebral [4-6].

\section{CONCLUSÃO}

A bitoracotomia ântero-lateral com esternotomia transversa proporciona intervenção cirúrgica em um único estágio, amplia o acesso à aorta torácica e permite correção total da afecção com maior rapidez, tornando-se um fator decisivo na boa evolução desses pacientes.

\section{REFERÊNCIAS BIBLIOGRÁFICAS}

1. Clouse WD, Hallet Jr JW, Schaff HV, Gayari MM, IIstrup DM, Melton LJ. Improved prognosis of thoracic aortic aneurysms: a population-based study. JAMA. 1998;280(22):1926-9.
2. Coady MA, Rizzo JA, Hammond GL, Mandapati D, Darr U, Kopf GS et al. What is the appropriate size criterion for resection of thoracic aortic aneurysm? J Thorac Cardiovasc Surg. 1997;113(3):476-91.

3. Kouchoukos NT, Masetti P, Rokkas CK, Murphy SF. Singlestage reoperative repair of chronic type A aortic dissection using the arch- first technique. Ann Thorac Surg. 2002;74(5):S1800-2.

4. Kouchoukos NT, Masetti P, Rokkas CK, Murphy SF, Blackstone EH. Safety and efficacy of hypothermic cardiopulmonary bypass and circulatory arrest for operations on the descending thoracic and thoracoabdominal aorta. Ann Thorac Surg. 2001;72(3):699-708.

5. Okita Y, Takamoto S, Ando M, Morota T, Yamaki F, Matsukawa $\mathrm{R}$ et al. Repair for aneurysms of the entire descending thoracic aorta of thoracoabdominal aorta using a deep hypothermia. Eur J Cardiothorac Surg 1997;12(1):120-6

6. Carrel TP, Berdat PA, Robe J, Gysi J, Nguyen T, Kipfer B, et al. Outcome of thoracoabdominal aortic operations using deep hypothermia and distal exsanguination. Ann Thorac Surg. 2000;69(3):692-5. 